\title{
A Self-Aware Epilepsy Monitoring System for Real-Time Epileptic Seizure Detection
}

\author{
Farnaz Forooghifar*, Amir Aminifar*, Leila Cammoun ${ }^{+}$, Ilona Wisniewski ${ }^{+}$, \\ Carolina Ciumas ${ }^{+}$, Philippe Ryvlin ${ }^{+}$, David Atienza* \\ ${ }^{*}$ Embedded Systems Laboratory, Swiss Federal Institute of Technology Lausanne (EPFL), Switzerland \\ ${ }^{+}$Department of Clinical Neuroscience, Lausanne University Hospital (CHUV), Switzerland \\ Email: \{farnaz.forooghifar, amir.aminifar, david.atienza\}@epfl.ch \\ Email: \{leila.cammoun, ilona.wisniewski, carolina.ciumas, philippe.ryvlin\}@chuv.ch
}

\begin{abstract}
Epilepsy is one of the most prevalent paroxystic neurological disorders that can dramatically degrade the quality of life and may even lead to death. Therefore, real-time epilepsy monitoring and seizure detection has become important over the past decades. In this context, wearable technologies offer a promising solution to pervasive epilepsy monitoring by removing the constraints with respect to time and location. In this paper, we propose a self-aware wearable system for real-time detection of epileptic seizures on a long-term basis. First, we propose a multi-parametric machine learning technique to detect seizures by analyzing both cardiac and respiratory responses to seizures, which are obtained using only the ECG signal. Second, in order to enable long-time epilepsy detection, we introduce the notion of self-awareness in our real-time wearable system. We evaluate the performance of our proposed solution based on an epilepsy database of more than 211 hours of recording, provided by the Lausanne University Hospital (CHUV), on the INYU wearable sensor. Our proposed system achieves a sensitivity of $88.66 \%$ and a specificity of $\mathbf{8 5 . 6 5 \%}$ before applying self-awareness. Moreover, by controlling the energy-quality trade-offs using our self-aware energy-management technique, we can tune the battery lifetime of the wearable system to last between 67.55 and 136.91 days while, still outperforming the state-of-the-art techniques for wearable seizure detection, by achieving from $85.54 \%$ to $79.33 \%$ geometric mean of specificity and sensitivity.
\end{abstract}

Index Terms-epileptic seizure detection, cardiac system, respiratory system, self-awareness, electrocardiography, heart-rate variability

\section{INTRODUCTION}

Neurological disorders contribute to $6.3 \%$ of the disabilityadjusted life years (i.e., time lost with respect to an ideal healthy life situation) among all categories of diseases [1]. Epilepsy is one of the most common neurological disorders affecting more than 65 million people worldwide [2]. Despite the recent advances in anti-epileptic drugs, one-third of epilepsy patients still suffer from seizures. More importantly, epilepsy represents the second neurological cause of years of potential life lost, primarily due to seizure-triggered accidents and sudden unexpected death in epilepsy (SUDEP) [3].

The possibility of monitoring epileptic patients in real time and on a long-term basis is poised to improve the quality of life and reduce the mortality rate in these patients [4], [5]. In particular, by using such real-time epileptic seizure

Manuscript received July 15, 2019. detection mechanisms, it is possible to notify family members, caregivers, and emergency units in case of a seizure. Therefore, it should be possible to reduce seizure-related injuries, status epilepticus, and SUDEP [6]. However, realtime epileptic seizure detection is not possible without energyefficient wearable technologies, which are also key enablers for long-term patient monitoring in ambulatory settings.

In this article, we adopt the notion of self-awareness to enable real-time long-term epilepsy monitoring. Self-awareness is introduced to a system, so that it can obtain knowledge about itself and also the environment in which it is operating. This information enables the system to monitor its performance, adapt to changes and improve autonomously (in terms of system's objectives, such as, power consumption and performance) [7], [8]. Self-awareness has two main phases of learning and reasoning, which are driven by the system's goal. This is translated to an Observe-Decide-Act (ODA) loop in self-aware systems, where a set of observations are done according to the system's goal. Then, a set of possible actions are determined and, finally, a decision is made about the best use of the available actions to meet the system's goals [9]. Self-awareness can be indeed the key to design the nextgeneration of intelligent medical wearable systems. To ensure extreme energy efficiency, having knowledge about self and environment is necessary for the system. Self-awareness can be applied in seizure detection systems by fulfilling three major properties: reflecting current situation, predicting the possibilities to improve energy reduction and adopting the right strategy in the system [10]-[12].

In the case of epilepsy monitoring, the gold standard to identify and adopt suitable strategies is based on the videoElectroencephalogram (EEG), which involves monitoring the brain electrical activity of patients using EEG, together with closed-circuit video observation. However, the long-term EEG monitoring outside the hospital premises using hats and caps can cause social stigmatization and discomfort for patients [13], due to their intrusive nature. Hence, there is currently no viable scalp-EEG-based solution for the very long-term (i.e. over weeks, months or years) ambulatory monitoring of epileptic seizures. On the other hand, intracerebral EEG monitoring can be performed over very long periods of time, but represents an invasive and expensive technology. Moreover, it can only be proposed to a small minority of 
patients [14].

Alternatives to scalp-EEG for non-invasive seizure detection derive from their impact on motor activity, reflected in 3Daccelerometry and electromyography (EMG) [15], [16], as well as from their interaction with the autonomic nervous system (ANS) [17], [18], with changes in both sympathetic and parasympathetic nervous activities [19]. These changes in ANS are reflected into changes in heart rate [20]-[26], respiratory function [27]-[31], and electrodermal response [32]. The most common cardiac change associated with seizures is ictal tachycardia, often exceeding $100 \mathrm{bpm} \mathrm{[33]-[35].} \mathrm{Seizures} \mathrm{can} \mathrm{also}$ affect respiratory function (e.g., tachypnea, hypopnea, apnea). Neurogenic pulmonary edema, central apnea and asystolia might also occur and can result in SUDEP [27]-[29]. A causal analysis of these cardiac and respiratory characteristics can only be performed by continuous monitoring of cardiorespiratory functions.

Currently, wearable devices used for non-EEG seizure detection can only reliably detect generalized tonic-cronic seizure (GTCS), for which the associated motor activity and ANS changes are both prominent and stereotyped. However, these seizures only represent a minority of epileptic attacks. There is thus a need to develop systems enable to identify the more variable and difficult to detect non-generalized focal seizures (FS). To this end, multi-parametric monitoring that includes cardiorespiratory biosignals might prove useful.

In this article, we extend our previous work [36] and propose a novel wearable system by combining multi-parametric biosignal processing and machine learning with the selfawareness notion. Real-time monitoring and detection of epileptic seizures is done based on the time series extracted from the ECG signal. Our monitoring system is capable of capturing the changes in real-time in both cardiac and respiratory functions due to seizures, by extracting a novel set of features, through detailed time- and frequency-domain analysis of the ECG signal. Moreover, in our self-aware monitoring system, the most energy-hungry tasks, such as complex signal processing, are performed only if necessary, by utilizing the self-awareness notion. As a result, the main contributions of this article are as follows:

- The first key contribution of this work is a reliable epileptic seizure detection system relying on wearable technology, which is based on cardiac and respiratory functions analysis using machine learning techniques, which include a new feature set that has not been used previously in the literature of epilepsy monitoring, and which significantly improves previous works in terms of detection quality. In addition, in this work we adopt a random forest algorithm, instead of the support vector machines, to obtain more robust results and avoid overfitting. In addition, by capitalizing on this new random forest algorithm, we are able to expose the energy-quality trade-off resulting from introducing the self-awareness concept in our proposed epilepsy monitoring wearable system. Furthermore, we validate our wearable system based on a dataset provided by Lausanne University Hospital (CHUV), which contains more than 211 hours of recordings for 18 epileptic patients, instead of the initial seven in our previous work, to rigorously evaluate the proposed technique. Overall, our proposed detection machine-learning technique achieves a sensitivity of $88.66 \%$ and a specificity of $85.65 \%$.

- The second key scientific innovation of this work is a novel energy-management scheme for medical wearable systems, which is based on the notion of self-awareness in order to realize real-time and energy-aware monitoring on a long-term basis. To reach our goal of improving the battery lifetime, the confidence of the low-complexity classifier of the system is being observed. Then, based on this information, the decision of whether to invoke the complex classifier is made. According to this decision, the action of switching between the two classifiers takes place. Our proposed technique is extensible because it can be applied to different health monitoring systems that use learning models for detection and prediction of health pathologies.

- We implement our proposed epilepsy monitoring approach, including this novel self-aware energymanagement technique, on the SmartCardia INYU wearable sensor [37] to demonstrate the real-time operation and evaluate its battery lifetime. We also explore the possible energy-quality trade-offs enabled by our new self-aware energy-management technique in order to demonstrate the extended battery lifetime of our wearable system from 67.55 to 136.91 days. Finally, we compare our proposed technique with state-of-the-art systems and show that our proposed technique outperforms the existing techniques by achieving $85.54 \%$ to $79.33 \%$ geometric mean (of specificity and sensitivity).

The rest of the article is organized as follows. In Section II, we review epileptic seizure detection techniques and existing wearable devices. In Section III, we present a high-level description of our system. Section IV describes our proposed features extraction procedure using times series. Section V describes our seizure detection machine learning technique and the energy management mechanism. Then, Section VI presents our experimental setup and Section VII describes our experimental results. Finally, in Section VIII, we summarize the main conclusions of this work.

\section{STATE OF THE ART}

The state-of-the-art technology for ECG-based seizure detection is that incorporated in the Vagus Nerve Stimulation (VNS) therapy device [38]. This uses a pre-thoracic implanted device to detect seizure-induced tachycardia and uses this information to trigger antiepileptic electrical stimulation of the left vagus nerve at the neck [39]. This represents an invasive and relatively expensive medical procedure, with some risks of adverse events, which is thus not appropriate for solely monitoring seizure in the majority of patients with epilepsy [40].

The gold standard in terms of non-invasive seizure detection is EEG monitoring, which has been used for decades in highly specialized and costful hospital environment (epilepsy monitoring unit). However, the necessity to permanently wear 
a cap and the high susceptibility of EEG recordings to artefacts as soon as patients are moving, has not yet enabled the use of EEG for very ambulatory chronic monitoring. To extract information from the EEG signal, several sets of features are used in the literature. These features include the wavelet transform [41], [42], entropies [42], [43], Hilbert marginal spectrum [44], fusion features [45], and tunable Q-factor [46], among other time-domain and frequency-domain features. In [47], the authors consider a combination of EEG and ECG monitoring to improve the results. To make collection and labeling of personalized EEG data easier in [48] a self-learning algorithm is used on new data.

Regarding non-invasive non-EEG based seizure detection devices, bed sensors [49], [50] have shown to be effective for detecting nocturnal convulsive seizures, but cannot monitor day-time seizures [51]. In addition, arm-band electromyography (EMG) and wrist-band devices [52], [53], which monitor either accelerometry or electrodermal activity, also proved reliable for detecting generalized tonic-clonic seizures (GTCS) with high sensitivity and acceptable false-alarm rates. However, it has been shown that they cannot detect other seizure types [54], [55]. Moreover, GTCS only represent approximately $15 \%$ of all epileptic seizures, stressing the need for other seizure detection devices.

In this respect, ECG-based detection of epileptic seizures has attracted a lot of attention. Heart rate fitting was used in [56], leading to a sensitivity of above $90 \%$ and a positive predictive value above $50 \%$, but with a trade-off between the sensitivity and positive predictive values that is not acceptable for medical use. Then in [57], the authors propose an approach that combines time-domain and frequency-domain features of heart-rate variability, but it is limited to the specific case of newborns, and it only reaches a sensitivity of $60 \%$ and a specificity of $60 \%$. In [58], using a similar approach, the authors achieve a sensitivity of $70 \%$ and a corresponding false-alarm rate of 2.11 per hour, which is still unacceptable for medical use in ambulatory patients. Finally in [59], a robust beat-detection algorithm was combined with a wireless real-time epileptic seizure detector. The proposed algorithm could detect successfully the seizures for only two out of three studied patients, which is a very small set to be able to draw statistically significant conclusions. In [60], the authors use peak-to-peak intervals and average heart-rate for epileptic seizure detection, but considering only three patients, which is also not sufficient to obtain conclusive results.

In [61], the authors propose a semi-automatic (i.e., requires manual intervention of medical experts) algorithm that considers peak-to-peak intervals and QRS-complex and uses several mathematical techniques, including cubic spline interpolation, principle component analysis, eigen-value decomposition, and clustering techniques. The authors achieved positive predictive values of $85.7 \%$ for focal seizures and $57.3 \%$ for GTCS. In [62], the authors proposed an approach to detect epileptic seizures, restricted to high-quality ECG signals, which are selected manually by expert visual analysis. Hence, such semi-automatic techniques cannot be adopted for monitoring patients in real time, using wearable systems. The proposed algorithm reached a false-negative rate of $14.5 \%$ with a high false-positive rate of 1.1 per hour. Alternatively, the proposed algorithm could be tuned to reach a better false-negative rate of only $2 \%$, but with an unacceptable false-positive rate of 9.5 per hour. This illustrates that such techniques cannot be readily applied in the context of real-time wearable systems, due to the complexity introduced by manual intervention of medical experts, thus the design of an entirely automatic seizure detection system represents a major challenge.

Another line of research in this topic has considered the use of Lorenz plots parameters as features for detection of epilepsy in [63], [64]. In these studies, epileptic seizures were detected with an accuracy of $76.47 \%$ for the target set of 17 patients. Nonetheless, this detection figure still does not fulfill the requirements for a reliable epilepsy detection system. Then, in order to improve the detection performance, in [65], the authors combined heart-rate variability and Lorenz-plot features and achieved a sensitivity of $94.1 \%$ and a false-alarm rate of 0.49 per hour. As it is shown in the experimental results (Section VII), despite improving the detection quality, these features do not result in acceptable detection performance, in comparison with our proposed features. Moreover, this work was performed in an offline setting (i.e., using a postprocessing analysis of the signal after seizures have already occurred), hence it cannot be adopted by real-time wearable systems. Finally, the authors also do not consider the energy consumption and battery lifetime of the proposed techniques, which is required for long-term real-time and ambulatory monitoring of patients.

We consider the application of self-awareness in the context of medical wearable systems. This is a new concept that has been considered recently for embedded systems to enable correct functionality within desired constraints, in spite of highly dynamic changes in the applications and the environment [66]-[71]. In particular, self-awareness has also been applied to biomedical systems, where the quality of the output of the system is significantly affected by different conditions of the patient as well as the environment. In [72], the authors adopt situation-awareness and personalized data (such as age, gender, etc.) to increase the accuracy of remote health monitoring. Furthermore, different priorities are given to the sensory data collection to consider the energy efficiency and dependability of the system. In [73], different parameters such as system's confidence are measured to improve the observation process and get high-quality description of the system from raw data. Therefore, although self-awareness is used in a wide range of systems, it has not been considered for detection of pathological health conditions up to this date.

In conclusion, previous studies in ECG-based epileptic seizure detection systems have mainly focused on heart-rate variability and Lorenz-plot features in controlled environments and offline settings, which dramatically limit its possible use in the context of real-time wearable medical systems. Also, in the literature of biomedical systems the concept of self-awareness has started to be explored, but never considering its use for energy-efficient designs of systems monitoring pathologies. Therefore, we take into account the limitation of such systems in terms of computational resources and energy to exploit the concept of self-awareness, while also considering its medical 


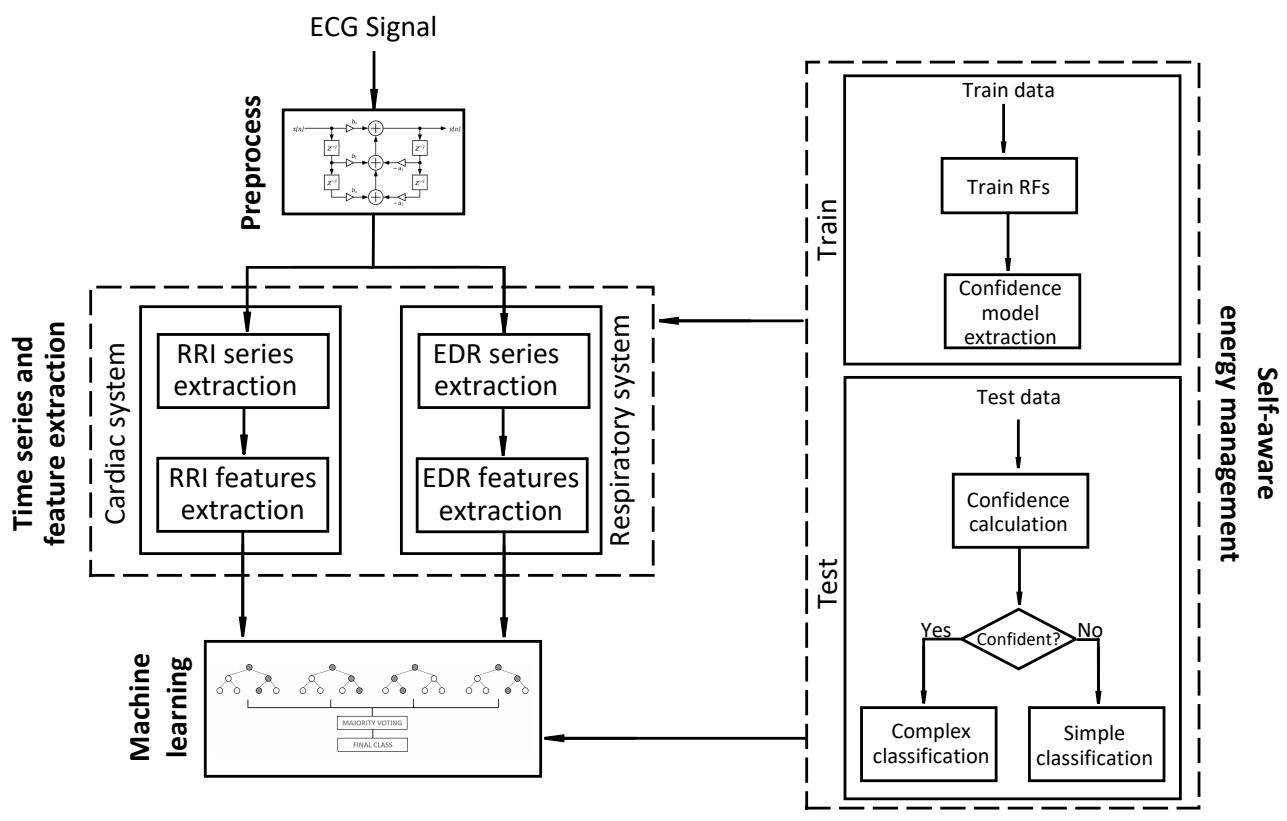

Figure 1: Overview of our proposed self-aware seizure detection system. The three main parts of our proposed system are highlighted by dotted lines.

impact on real-time seizure detection based on both cardiac and respiratory systems.

\section{Self-Aware Seizure Detection System DESCRIPTION}

In this section, the main architectural building blocks of our proposed system are described. A high-level overview of our real-time wearable seizure detection system is shown in Figure 1. The details of our seizure detection machine learning technique and energy-management strategy are explained in Sections IV and V.

\section{A. Cardio-Respiratory Time Series Analysis and Features Ex- traction}

In this paper, as the first step, the frequencies above $60 \mathrm{~Hz}$ are removed. Then, two time series, R-peak to Rpeak Interval (RRI) and ECG-Derived Respiration (EDR), are extracted from the ECG signal. In particular, we extract features to capture the distinctive patterns of epileptic seizures in both physiological subsystems of humans. First, heart-rate variability (HRV), Lorenz plot and multifractality features are considered to capture the variations and fluctuations in the time intervals among the heartbeats based on the RRI time series. Second, the frequency content and the irregularity of EDR are captured by using the Plomb transform and the entropy features, respectively. The details of our proposed approach using these two time series, together with the corresponding features, are provided in Section IV.

\section{B. Self-Aware Learning and Energy Management}

To detect epileptic seizures in real-time with wearable devices, we adopt the random forest algorithm [74], considering the features extracted from the RRI and EDR time series. Random forest shows not only high performance in terms of the accuracy of detecting seizures, but it is also suitable to be implemented on resource-constrained wearable embedded systems, as we show in Section VII.

In addition, as real-time detection of seizure is not possible without long-term monitoring systems, in order to maximize the battery lifetime, we adopt the notion of self-awareness to achieve energy efficiency and quality-scalability and enable long-term epilepsy monitoring. In particular, we develop a selfaware seizure detection technique where classification can be done with either a simple set of features or a more complex set. In fact, the entire set of features are not used for seizure detection unless confident classification based on the set of simple features is not possible. Then, we take advantage of the multi-mode execution possibilities of the platform, in a selfaware fashion, so that the energy consumption is reduced while quality of system remains in an acceptable level for medical use. Moreover, our system is kept in an ultra-low power (energy-saving) mode when tasks terminate their executions. A detailed description of our self-aware energy-management mechanism is provided in Section V-B.

\section{Cardio-Respiratory Time Series Analysis and FEATURE EXTRACTION PHASE}

In this section, we detail how we capture the cardiac and respiratory responses by extracting two time series and subsequently obtaining the relevant features from them. 


\section{A. Cardiac System Analysis for Epileptic Seizures Detection}

First, we extract the RRI time series and features from the ECG signal to capture the cardiac function.

1) RRI Time Series Extraction: On a normal ECG, there are typically up to three visible components: the $\mathrm{P}$ wave, QRS complex (Q, R, and S), and T wave. The R-peak is the most straightforward characteristic point to identify and corresponds to ventricular depolarization. Accordingly, the $\mathrm{R}$-wave is distinguished by the peaks of the ECG signal. Therefore, the distance between two successive R-peaks is captured by RRI time series. In this paper, we adopt the PanTompkin algorithm [75] to detect the R-peaks and RRIs.

Guaranteeing high-quality features requires extraction of the correct locations of the R-peaks. However, the delineation algorithm may misdetect the peaks due to poor signal quality and noise. Therefore, we remove these incorrect values based on the Thompson-tau method [76], which is a statistical algorithm that detects the outliers in the RRI time series.

2) RRI Feature Extraction: In order to capture the cardiac function, the heart-rate variability (HRV), we extract Lorenz plot and multifractality features from the RRI time series. These features are chosen in order to reflect the changes in cardiac system such as ictal tachycardia and bradycardia, which, as discussed before, have direct impact on variability in RR intervals. The HRV features we include are the following ones:

- MeanNN: The mean of R-R intervals.

- SDNN: The standard deviation of R-R intervals.

- RMSSD: The root mean square of difference between adjacent R-R intervals.

- Total Power (TP): The variance of R-R intervals.

- NN50: The number of pairs of adjacent R-R intervals whose difference is greater than 50 msecs.

- LF: The power in the low-frequency band $(0.04 \mathrm{~Hz}-$ $0.15 \mathrm{~Hz}$ ) of RRI. The power in the low-frequency band reflects both the sympathetic and parasympathetic activity of the nervous system [63].

- HF: The power in the high-frequency band $(0.15 \mathrm{~Hz}-$ $0.4 \mathrm{~Hz}$ ) of RRI. The power in the high-frequency band reflects the parasympathetic nervous system activity [63].

- LF/HF: The ratio of LF to HF. This feature captures the balance between the sympathetic nervous system activity and the parasympathetic nervous system activity.

The second set of features we extract from the RRI time series is the Lorenz plot features [63]. Lorenz plot (or Poincare plot) is the process of plotting $R R I(n)$ against $R R I(n+1)$. Lorenz plot can capture the dynamic variation of the RR intervals. These dynamics can be captured by extracting several statistical properties from the Lorenz plot, as follows:

$$
\begin{array}{r}
S d 1=\sigma\left(\frac{\sqrt{2}}{2}[R R I(1)-R R I(2), R R I(2)-R R I(3)\right. \\
, \ldots, R R I(k)-R R I(k+1)]), \\
S d 2=\sigma\left(\frac{\sqrt{2}}{2}[R R I(1)+R R I(2), R R I(2)+R R I(3)\right. \\
, \ldots, R R I(k)+R R I(k+1)]),
\end{array}
$$

where $\sigma$ denotes the standard deviation of the time series. The Lorenz plot features are the following ones:

- T: The transversal length is defined as $T=4 \cdot S d 1$.

- L: The longitudinal length is defined as $L=4 \cdot S d 2$.

- CSI: The cardiac sympathetic index is defined as $C S I=$ $\frac{L}{T}$.

- MCSI: The modified cardiac sympathetic index is defined as $M C S I=\frac{L^{2}}{T}$ and emphasizes the longitudinal value $L$, which increases during the pre-ictal and early ictal phase [63].

- CVI: The cardiac vagal index is defined as $C V I=$ $\log _{10}(L \cdot T)$.

The final set of features are multifractality ones, which are extracted to reflect the fluctuation of RRI time series and analyze more complex irregularities in heart-rates [77]. Monofractal signals are homogeneous because they have the same scaling properties throughout the entire signal. Multifractal signals, on the other hand, can be decomposed into many subsets characterized by different local Hurst exponents $h$. Then, the statistical properties of the different subsets characterized by these exponents $h$ can be quantified by the function $D(h)$ :

$$
D(h)=q h-\tau(q) .
$$

The function $\tau(q)$ is calculated as shown in Equation (4), where $Z_{q}(a)$ is the partition function that is defined as the sum of the $q^{\text {th }}$ powers of the local maxima of the modulus of the wavelet transform coefficients at scale $a$ :

$$
Z_{q}(a) \approx a^{\tau(q)} .
$$

In [77], the authors show that during the normal heart function we have significant value for $D(h)$ for $0.05<h<0.27$, while in heart failure this function is centered near $h \approx 0.22$ with a very small radius. To capture the fluctuations in RRI time series, we have chosen $D(h)$ for different values of parameter $h$ as features, in the aforementioned interval.

\section{B. Respiratory System}

In this section, we detail the extraction of the EDR time series and features from the ECG signal in order to capture the respiratory function.

1) EDR Time Series Extraction: Respiration is a physiological function that is modulated by the autonomic nervous system (ANS). Then, the respiratory signal is typically recorded by spirometry, pneumography, or plethysmography. However, it is also possible to extract respiratory signals from the ECG signal, which is known as the EDR signal [78]. Indeed, ECGderived respiratory methods exploit the respiratory-induced changes of the ECG to provide an alternative respiratory signal. In this paper, we adopt the algorithm proposed in [78] for EDR extraction. Thus, after removing the baseline wander from the ECG signal, the R-peaks are located on the preprocessed signal $\left(E C G_{\text {filtered }}\right)$ and we obtain the EDR by calculating the area enclosed in the regions that are $100 \mathrm{msec}$ beyond the R-peaks, as follows: 


$$
E D R(n)=\sum_{k=R_{p}(n)-100 m s e c}^{R_{p}(n)+100 m s e c}\left|E C G_{\text {filtered }}(k)\right|,
$$

where $R_{p}$ is the location of the R-peaks described in Section IV-A1.

2) EDR Feature Extraction: We extract two different sets of features from the EDR time series. First, we extract features related to different entropies, which are used to capture the non-stationary and non-linearity characteristics of the EDR signal. Then, we apply a discrete waveform transform (DWT) by decomposition of the EDR time series to level seven as a preprocessing step to improve entropy features quality [79]. Hence, in this paper the following entropies are used as features:

- Sample entropy: Given a time series $X=x(1), \ldots, x(N)$, sequences of length $m\left(X_{m}(i)\right)$ are extracted [80]. Two patterns $X_{m}(i)$ and $X_{m}(j)$ are similar if the difference between any pair of corresponding measurements in the patterns is less than $r$ :

$$
|X(i+k)-X(j+k)|<r, \forall k=[0, m) .
$$

The sample entropy is defined as:

$$
\operatorname{SampEn}(x, m, r)=\ln \left(\frac{C_{m}(r)}{C_{m+1}(r)}\right),
$$

where $C_{m}(r)$ is the number of patterns in $X_{m}$ that are similar to $X_{m}(i)$, excluding self-matches, after normalization. In this work, we use $m=2$, and $r=$ $k$.std(signal), where $k \in\{0.2,0.35\}$, which are chosen according to the experiments in Section VII.

- Permutation entropy: Given a time series $\left\{x_{t}\right\}_{t=1, \ldots, T}$, where $\mathrm{T}$ is the length of the time series, all possible $n$ ! permutations are calculated [81]. There are two parameters defined: parameter $\pi$ which corresponds to the permutation type, and parameter $n$ which represents the number of instances considered in order to estimate the permutation entropy.

$$
p(\pi)=\frac{\text { number of perms that have the type } \pi}{T-n+1} .
$$

The permutation entropy of order $n \geq 2$ is defined as:

$$
P E(n)=-\sum_{\pi} p(\pi) \log (p(\pi)) .
$$

- Renyi entropy: This entropy is calculated as proposed by [82]:

$$
R E(q)=\frac{1}{1-q} \ln \sum p_{i}{ }^{q},
$$

where $q \neq 1$ and $p_{i}$ defines the total spectral power in the i-th band.

- Shannon entropy: This entropy is the special case of Renyi entropy for $q=1$, namely:

$$
S E=-\lim _{q \rightarrow 1} R E(q)=-\sum p_{i} \ln \left(p_{i}\right) .
$$

- Tsallis entropy, which is defined as follows [82]:

$$
T E(q)=\frac{1}{1-q}\left(1-\sum p_{i}{ }^{q}\right) .
$$

The second set of features, extracted from the EDR time series, captures the power spectral density (PSD). However, the EDR time series is unevenly sampled because it depends on the varying heartbeats. Therefore, conventional spectral analysis techniques, which assume uniformly-sampled signals, cannot be used to extract the spectral content of the EDR time series. Consequently, we use the Plomb transform to estimate the PSD of non-uniformly sampled signals [83], [84]. This transformation is based on the Lomb-Scargle method [83], [84], which does not require resampling or interpolation and works directly with the non-uniform sampled signals. Thus, in our work, we adopt the Plomb transform to estimate the PSD of the EDR time series. In this work the spectrum of EDR is divided into several sub-bands and the power of these sub-bands are used as features.

\section{Self-Aware Learning And Energy Management}

In this section, we present our new self-aware energymanagement technique to enable real-time and long-term epilepsy monitoring in ambulatory settings, while still maintaining the reliability required in the medical domain. Also, we introduce the notion of self-awareness in our epileptic seizure detection system to improve its battery lifetime and energy efficiency.

\section{A. Machine Learning Algorithm}

In our proposed self-aware wearable system, we adopt the random forest classifier as the learning technique for the classification of non-seizure (inter-ictal) and seizure (ictal) segments.

Decision trees are tree-like structures in which each internal node represents a decision on a feature, each branch represents the outcome of the decision, and each leaf node represents a class label (decision taken after computing all attributes). Bagging (bootstrap aggregation) is an ensemble learning technique that is used for reducing the variance of a statistical learning method. It reduces the variance by averaging over the output of a set of $n$ independent observations $Y_{1}, Y_{2}, \ldots, Y_{n}$, each with variance $\sigma^{2}$. The variance of the mean $\bar{Y}$ of the observations is given by $\frac{\sigma^{2}}{N}$. Given a training set $D$ of size $m$, bagging generates $N$ new training sets $D_{i}$, each of size $m^{\prime}$, by sampling from $D$ uniformly and with replacement.

Hence, $N$ different bootstrapped training sets are subsampled from the single dataset and used to train a collection of $N$ decision trees, where every tree is trained only on one of the subsampled training dataset. In testing new features, the decision on the seizure/non-seizure class is made by majority voting on the output predicted by each one of the $N$ trees. The value of $N$ is chosen to be 100 in this paper to have high quality detection and at the same time acceptable energy consumption, as the complexity of the online classification using this classifier depends only on number of bags used in bootstrap aggregation. Therefore, we reduce the complexity 


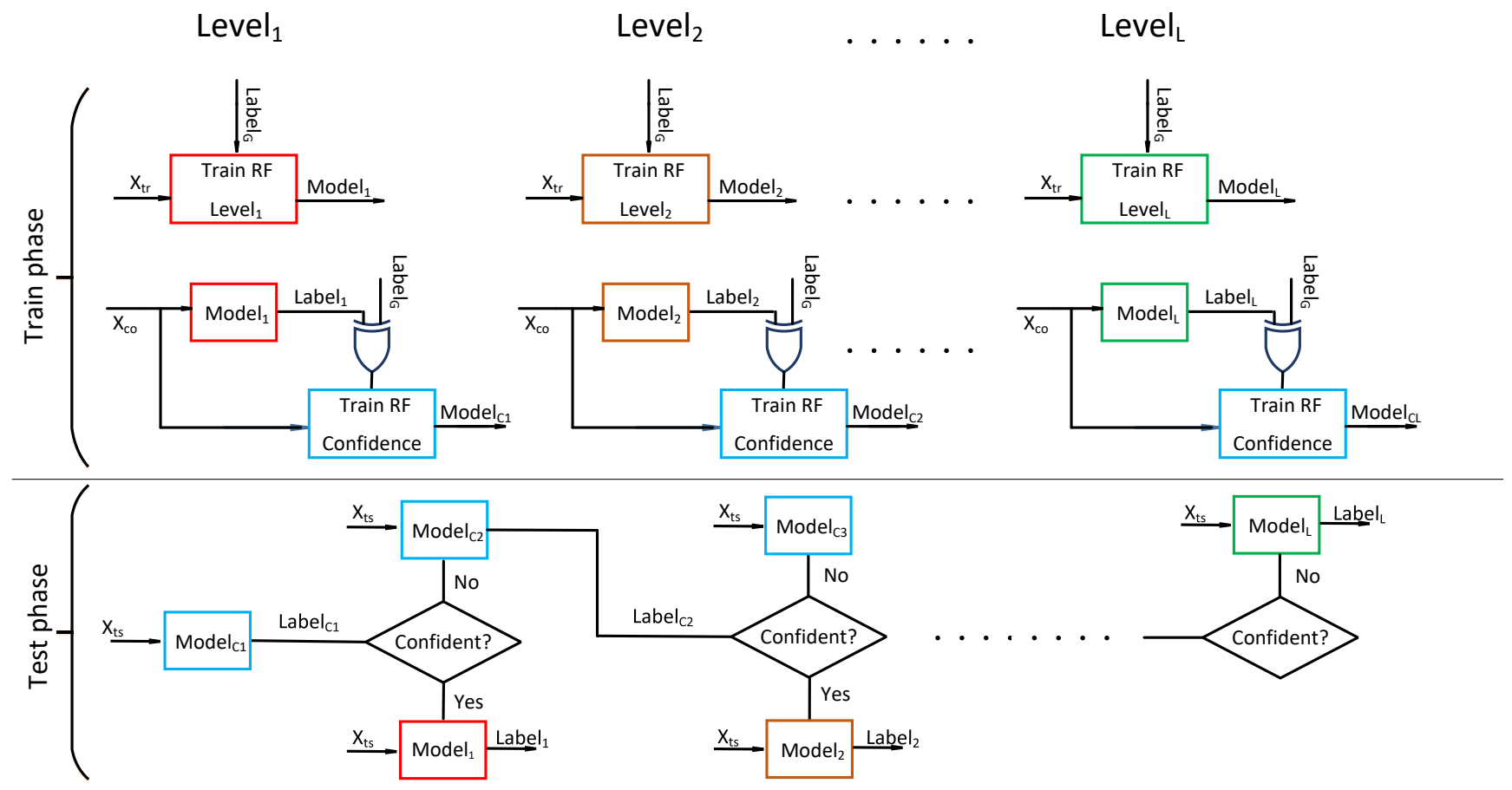

Figure 2: Self-aware classification to improve energy efficiency. In the training phase, the classifiers and confidence models are generated. Then, in the test phase, after calculating the confidence at each level, the level of the classifier to use is decided.

of implementation on resource-constrained medical wearable platforms.

\section{B. Self-Aware Energy Management}

To detect epileptic seizures, often only a few prominent features, which capture the cardiorespiratory response to epileptic seizures, are sufficient for confident classification. However, these few prominent features do not always cover the whole spectrum of bio-markers reflecting seizures. In this section, to keep the high detection performance, while improving the energy efficiency, we propose a self-aware classification technique.

The main idea of our self-aware energy-management technique is to use a simpler classification, with less features, whenever the results are deemed reliable and, otherwise, a more complex classification, with the full set of features. On the one hand, the simple classifier, while energy-efficient, cannot always provide high detection performance. On the other hand, the complex classifier, while providing high detection performance, cannot provide high energy-efficiency. This technique can be generalized to multiple levels of classifiers which differ in terms of detection performance and energy consumption. In this case we start with the simplest classifier with minimum energy consumption and detection performance. If the result is not deemed reliable, we switch to the next classifier and continue this procedure until the system is confident about its decision. Using this multilevel approach, we can also control the trade-off between the detection performance and the energy consumption according to the constraints of the application. The overall flow (both the train and test phases) of our self-aware classification technique is shown in Figure 2.

The training phase of our classification procedure is shown in Algorithm 1. In this procedure, first, we train the classifier models which includes detection models of different levels (Model $)_{l}$ ) based on the first part of the train data $\left(X_{t r}\right)$ and the corresponding ground-truth labels $\left(\right.$ Label $\left._{G}\right)$ (Line 3$)$. Then, we use the generated models to predict the labels corresponding to the second part of train data $\left(X_{c o}\right)$ (Line 4). For each level, the ground-truth labels and the predicted ones $\left(\right.$ Label $\left._{l}\right)$ are compared and the comparison result is used to train another RF classifier (Line 5). This classifier also uses the level $_{l}$ set of features and generates a model $\left(\mathrm{Model}_{\mathrm{Cl}}\right)$ that captures the confidence in classification based on the level $_{l}$ classifier.

This procedure is not restricted to the class of the random forest algorithm and can be applied considering any binary classification technique. The random forest is chosen as it achieves a higher quality with respect to the SVM classification used in [36]. The random forest method adopted in this work provides more robust results and avoids overfitting, due to bootstrap aggregating techniques. Moreover, the random forest algorithm has a special property that is very convenient in the context of self-aware systems. In particular, the random forest algorithm provides a confidence metric, based on the number of individual decision trees that agree in their decisions, which can be considered to explore the energy-quality trade-offs in our proposed epilepsy detection system.

The test phase of our classification procedure is shown in 

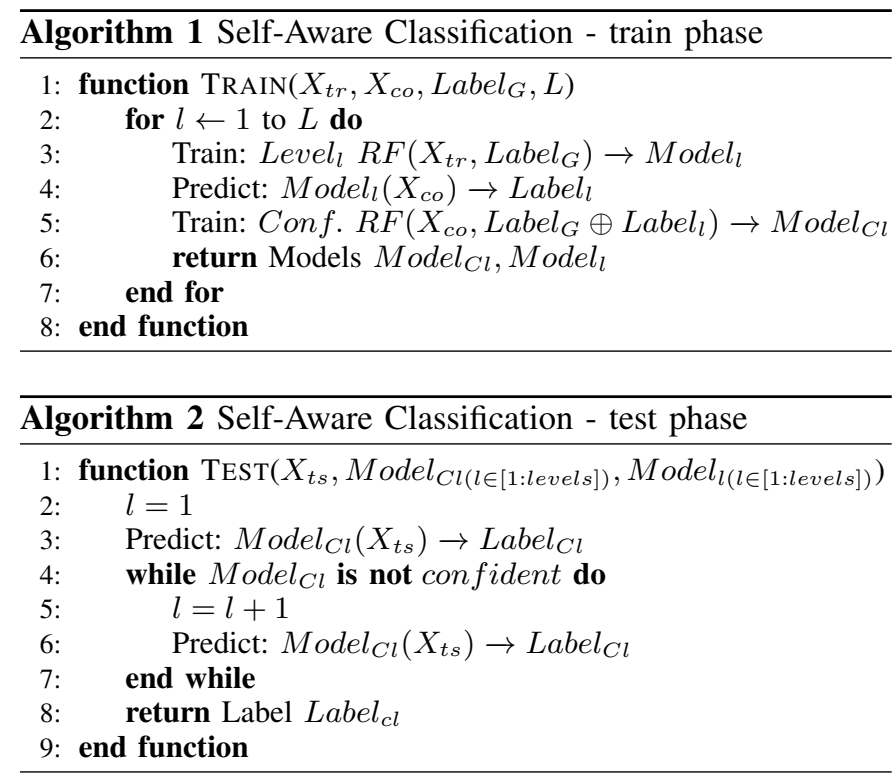

Algorithm 2. Based on the test data $\left(X_{t s}\right)$ and the confidence models $\left(\right.$ Model $\left._{C l}\right)$, the system is aware whether the level set of features can perform confident classification of the test data (Line 3). While the classification based on the level $_{l}$ set of features is not confident $\left(\right.$ Label $\left._{C l}\right)$, the number of levels is increased (Line 5). We continue predicting the confidence label $\left(\right.$ Label $\left._{C l}\right)$ and checking if the result is confident until the confidence is reached for a certain number of levels (Line 4-7).

In our proposed energy-management technique for the seizure detection system we have used two levels of classifiers and, as a result, the energy consumption of the system is also divided into two parts: (1) the level $_{1}$ classification, where the reduced set of features which are: meanNN, SDNN, RMSSD, total power, and NN50 from HRV features plus Lorenzplot features are sufficient, and (2) the level $_{2}$ classification, where all features are considered. Based on the probability of invoking the first level classifier, the total execution time of our wearable system can be calculated as follows:

$$
T_{\text {execution }}=T_{C}+P_{1} \cdot T_{1}+\left(1-P_{1}\right) \cdot T_{2},
$$

where $T_{\text {execution }}$ is the total execution time of our selfaware classification technique, $P_{1}$ is the probability of invoking the level $_{1}$ model (depends on the dataset), and $T_{C}, T_{1}$ and $T_{2}$ are the execution times of the confidence calculation, level $_{1}$ classification, and level $_{2}$ classification, respectively.

Finally, while here we describe our self-aware classification technique for only two levels, the proposed technique is not limited in the number of levels and can be generalized to any number of layers, as mentioned before. If $n$ is the number of levels and $P_{i}$ and $T_{i}$ are the probability (percentage) and execution time of $i$-th level occurrence, respectively. Then, the expected execution time is calculated as follows:

$$
T_{\text {execution }}=T_{C}+\sum_{i=1}^{n} P_{i} \cdot T_{i}
$$

which shows that the higher the probability of the simpler classifiers is (which can depend on the dataset), the lower the execution time of the entire system. Also as $n$ increases, we will have a more complex confidence measurement part and, as a result, $T_{C}$ (which is the sum of all confidence measurement parts at different levels) will increase.

\section{EXPERIMENTAL SETUP}

\section{A. Clinical Dataset}

Our proposed system was tested based on a dataset provided by the Lausanne University Hospital (CHUV). Data from 18 patients with epilepsy contributed to this dataset. Patients underwent in-hospital recording of their seizures for diagnostic purposes, and provided informed consent for their data to be re-used for research. Seizure recordings were performed using standard video-electroencephalography (EEG) together with single lead thoracic ECG. The latter is being used to ensure appropriate control of vital signs during seizure monitoring, and was acquired at a sampling rate of $256 \mathrm{~Hz}$. Video-EEG recordings were reviewed by a medical expert in epilepsy to annotate the onset and offset of all epileptic seizures. Annotated ECG data were then extracted and fully anonymized in order to be processed in this study. Overall, 211 hours of recordings were made available for this study, including 154 focal seizures.

During these in-hospital epilepsy monitoring periods, although patients are restraint to a hospital room, the clinical protocol allows them to circulate freely in the room and perform daily activities, i.e., walk around, sit at the table, eat meals, play cards, change clothes, and even certain do physical activities, e.g., ride stationary bikes. Therefore, a certain level of motion artifacts are already present in these signals. Consequently, as mentioned in Section III-A, we apply noise filters in the preprocessing phase of our proposed technique to remove the different types of noise, including motion artifacts, muscle artifacts, baseline wander and powerline interference. Nevertheless, while we have not analyzed the performance of our proposed technique in the presence of intense physical activities, it is probable that the quality of our proposed seizure detection technique degrades in such extreme cases and further studies are needed.

\section{B. Performance Metrics}

We use the leave-one-out method to evaluate the performance of our proposed self-aware system, where out of $K$ recordings, one is used as the test data and the other $K-1$ recordings are used as train data. This process is repeated to test all the recordings. In other words, the recording to be tested is left out and the model is trained on the remaining ones. The performance of the proposed algorithm is evaluated by measuring the specificity $(S p e c)$, sensitivity ( $S e n)$, and the geometric mean (gmean), which are defined as follows:

$$
\begin{aligned}
& \text { Spec }=\frac{T N}{F P+T N}, \\
& \text { Sen }=\frac{T P}{T P+F N},
\end{aligned}
$$




$$
\text { gmean }=\sqrt{\text { Spec } \cdot \text { Sen }}
$$

where $F P, T N, T P$ and $F N$ definitions are the following ones:

- False positive $(F P)$ : The patient is in the inter-ictal state, but the sample is classified as ictal.

- True negative $(T N)$ : The patient is in the inter-ictal state, and the algorithm declared that.

- True positive (TP): The patient is in the ictal state, and the algorithm detected that.

- False negative $(F N)$ : The patient is in the ictal state, and the sample is not classified correctly.

The geometric mean gmean is adopted since its high values reflect that both specificity (Spec) and sensitivity (Sen) are high, which is equal to having high quality detection. Conversely, if the geometric mean gmean is low, it means that Spec, Sen, or both are low, which is undesirable. Finally, we include the geometric mean as it is the only correct average of normalized measurements according to [85].

\section{Comparisons with State-of-the-Art}

We evaluate the efficiency of our wearable system against two state-of-the-art techniques, regarding seizure detection performance and energy efficiency.

The first technique [63] is based on the Lorenz plot and uses the features discussed in Section IV-A2. This approach reach an individual detection sensitivity of $100 \%$ for 13 out of 17 patients while the total coverage is $74.4 \%$ ( 35 out of 47 seizures).

In the second technique [65], seven features of HR including SDNN, RMSSD, LF, HF, SampEn (sampled entropy), CSI and CVI are used. In this approach, the authors use the SVM classifier with a Gaussian kernel for clinical data from 12 patients. The result is a sensitivity of $94.1 \%$ and a falsepositive rate of 0.49 per hour and uses an offline setting without considering energy consumption and battery life-time constraints.

\section{Implementation Platform}

The target hardware platform for our system is the SmartCardia INYU wearable sensor [37]. In this device, a singlelead ECG is recorded with the operating frequency between $250 \mathrm{~Hz}$ and $1 \mathrm{kHz}$. The device includes an ARM Cortex-M3 chipset (STM32L151RDT6) [86] for data analysis and classification, which is a low-power 32-bit microcontroller with 48 $\mathrm{kB}$ RAM and $384 \mathrm{kB}$ flash storage, and a maximum frequency of $32 \mathrm{MHz}$. This processor has several power-management modes, including active and sleep modes, with the possibility of dynamically switching between them. The INYU device is powered by a $710 \mathrm{mAh}$ battery. The ECG signal is acquired using silver-chloride electrodes for impedance pneumography [87]. The analog-to-digital converter (ADC) is the ADS7142 module [88], which is an event-driven ADC. This ADC has a power consumption of $900 \mathrm{nW}$ and works with a $0.5 \mathrm{uA}$ current.
Table I: Comparison among the quality of our proposed seizure detection system and the state-of-the-art techniques.

\begin{tabular}{|c|c|c|c|c|}
\hline Method & Confidence \% & Spec \% & Sens \% & Gmean \% \\
\hline Lorenz plot [63] & & 62.51 & 62.28 & 62.40 \\
\hline HRV + Lorenz plot [65] & & 74.44 & 76.03 & 75.23 \\
\hline \multirow{3}{*}{ Our system (SAEM) } & 60 & 78.28 & 80.40 & 79.33 \\
\cline { 2 - 5 } & 70 & 81.23 & 81.05 & 81.14 \\
\cline { 2 - 5 } & 80 & 81.99 & 85.42 & 83.69 \\
\cline { 2 - 5 } & 90 & 83.59 & 87.54 & 85.54 \\
\hline Our system (WOEM) & \multicolumn{3}{|c}{} \\
\hline
\end{tabular}

\section{EXPERIMENTAL RESULTS}

In this section, we evaluate the efficiency of our proposed wearable system against state-of-the-art techniques, both in terms of seizure detection performance and in terms of energy efficiency. To demonstrate the effectiveness of our self-aware energy-management technique, in addition to considering our final proposed system with self-aware energy management (referred to as SAEM), we also consider our system without any energy management (referred to as WOEM).

\section{A. Detection Performance}

In Table I, we assess the performance of the new wearable system against the state-of-the-art techniques [63], [65]. Our WOEM system achieves a sensitivity of $88.66 \%$, a specificity of $85.65 \%$, and a geometric mean of $87.15 \%$. First, our results show that epileptic seizures can be detected effectively by monitoring the ECG signal. Second, WOEM outperforms the approach in [63] by $24.75 \%$ and in [65] by $11.92 \%$, in terms of gmean related to detection performance. This demonstrates the importance of the respiratory system in epileptic seizure detection.

We also investigate the impact of our self-aware classification and energy management on the detection performance of our system. Table I shows that the proposed SAEM can achieve different qualities according to the confidence percentage, which relates to invoking the full classifier or not. In fact, it shows the minimum number of trees (out of 100) that should agree on using the simple classifier. By increasing this percentage, the simple classifier is used less frequently, and as a result, the quality is increased. However, a higher threshold increases the energy consumption (cf. Section VII-B). As we increase the threshold to $90 \%$ by steps of $10 \%$, the gmeans of $81.14 \%, 83.69 \%$ and $85.54 \%$ are achieved, which are $5.91 \%$, $8.46 \%$ and $10.31 \%$ higher than [65], respectively. Therefore, our proposed system is better than any existing real-time wearable seizure detection system. Moreover, it reaches an acceptable detection quality for long-term medical monitoring setups.

\section{B. Energy Efficiency}

To calculate the energy figures of our system, the random forest model parameters, which are calculated during the offline training phase of Section V-A, are loaded and stored in the $48 \mathrm{kB}$ RAM of the microcontroller of our system. To calculate the power consumption of the system, we consider 
Table II: Comparison among the battery lifetime of our proposed approach with (SAEM) and without (WOEM) energy management and the state-of-the-art techniques.

\begin{tabular}{|c|c|c|c|}
\hline Method & Execution part & Duty cycle \% & Lifetime (days) \\
\hline \multirow{4}{*}{ Lorenz [63] } & Delineation & 0.17 & \multirow{4}{*}{257.79} \\
\hline & Decompression & 0.57 & \\
\hline & Processing & 0.18 & \\
\hline & System & 0.92 & \\
\hline \multirow{4}{*}{ HRV + Lorenz [65] } & Delineation & 0.17 & \multirow{4}{*}{257.39} \\
\hline & Decompression & 0.57 & \\
\hline & Processing & 0.19 & \\
\hline & System & 0.93 & \\
\hline \multirow{5}{*}{$\begin{array}{l}\text { Our system (SAEM) } \\
60 \% \text { confidence }\end{array}$} & Delineation & 0.17 & \multirow{5}{*}{136.91} \\
\hline & Decompression & 0.57 & \\
\hline & Level 1 classification & 0.93 & \\
\hline & Level 2 classification & 5.46 & \\
\hline & System & 1.90 & \\
\hline \multirow{5}{*}{$\begin{array}{l}\text { Our system (SAEM) } \\
90 \% \text { confidence }\end{array}$} & Delineation & 0.17 & \multirow{5}{*}{67.55} \\
\hline & Decompression & 0.57 & \\
\hline & Level 1 classification & 0.93 & \\
\hline & Level 2 classification & 5.46 & \\
\hline & System & 4.01 & \\
\hline \multirow{4}{*}{ Our system (WOEM) } & Delineation & 0.17 & \multirow{4}{*}{50.15} \\
\hline & Decompression & 0.57 & \\
\hline & Processing & 4.72 & \\
\hline & System & 5.46 & \\
\hline
\end{tabular}

the current drawn in each processing mode and the duty cycle of the process. The signal processing and detection algorithms are executed every 60 seconds $\left(T_{\text {window }}\right)$. Thus, to calculate the duty cycle, we consider the time of the whole procedure ( $\left.T_{\text {execution }}\right)$, as follows:

$$
\text { DutyCycle }=\frac{T_{\text {execution }}}{T_{\text {window }}} .
$$

During the process, including delineation, feature extraction, and classification, the current is $10.5 \mathrm{~mA}$. For the rest of the time, the processor is put into the sleep mode and consumes $0.018 \mathrm{~mA}$. The ADC is active during the entire process and, as it is an event-driven converter, it draws a current of $0.5 \mathrm{uA}$.

The time complexity and battery lifetime of our proposed system using the INYU device are compared to previous studies [63], [65] in Table II. The two last methods in this table are our proposed system before (WOEM) and after (SAEM) applying our self-aware energy-management technique. The processing time SAEM is calculated based on Equation (13) and the probability of invoking the simple classifier, which is decreased from $78.48 \%$ to $32.05 \%$ as the confidence threshold is increased from $60 \%$ to $90 \%$. As a result, the battery lifetime of our system is increased to 136.91 days, compared to 50.15 days without any energy management. This demonstrates the effectiveness of our self-aware energy-management technique.

Finally, we compare the quality-lifetime trade-off of stateof-the-art and our proposed systems The results demonstrate that, as shown in Table II, our SAEM technique clearly outperforms state-of-the-art techniques in terms of detection performance, while still running for more than 136 days.

\section{CONCLUSION}

In this article, we have proposed a novel medical wearable system for long-term epilepsy monitoring and real-time detection of epileptic seizures, based on the cardiorespiratory function. We validated the detection performance and battery lifetime of our solution based on a multi-patient epilepsy dataset. Our proposed epilepsy detection wearable system achieves a sensitivity of $88.66 \%$, a specificity of $85.65 \%$, and a geometric mean of $87.15 \%$. This proposed system has been implemented using the state-of-the-art SmartCardia INYU multi-parametric sensor hardware to show its systemlevel real-time operation and energy scalability. Finally, by using our event-driven energy-management method a battery lifetime of 67.55-136.91 days is reached with reduction of only $1.61 \%-7.82 \%$ in detection performance.

\section{ACKNOWLEDGEMENTS}

This work has been partially supported by the MyPreHealth research project (Hasler Foundation project No. 16073), the WiTNESS project (Promobilia Foundation project No. 18079), and the Human Brain Project (HBP) SGA2 (GA No. 785907). The authors also would like to thank M. Nassralla for an initial implementation of a subset of the seizure detection features for epileptic signals, which was revised and extended to define the final set of used features.

\section{REFERENCES}

[1] W. H. Organization, Neurological disorders: public health challenges. World Health Organization, 2006.

[2] - (2016) Epilepsy. [Online]. Available: https : //www.who.int/mental_health/neurology/epilepsy/en/

[3] D. J. Thurman, D. C. Hesdorffer, and J. A. French, "Sudden unexpected death in epilepsy: assessing the public health burden," Epilepsia, vol. 55, no. 10, pp. 1479-1485, 2014.

[4] J. van Andel, R. D. Thijs, A. de Weerd, J. Arends, and F. Leijten, "Noneeg based ambulatory seizure detection designed for home use: what is available and how will it influence epilepsy care?" Epilepsy \& Behavior, vol. 57, pp. 82-89, 2016.

[5] P. Ryvlin, C. Ciumas, I. Wisniewski, and S. Beniczky, "Wearable devices for sudden unexpected death in epilepsy prevention," Epilepsia, vol. 59, pp. 61-66, 2018.

[6] A. Van de Vel, K. Cuppens, B. Bonroy, M. Milosevic, K. Jansen, S. Van Huffel, B. Vanrumste, L. Lagae, and B. Ceulemans, "Non-eeg seizure-detection systems and potential sudep prevention: state of the art," Seizure-European Journal of Epilepsy, vol. 22, no. 5, pp. 345-355, 2013.

[7] S. Kounev, J. O. Kephart, A. Milenkoski, and X. Zhu, Self-Aware Computing Systems, 1st ed. Springer Publishing Company, Incorporated, 2017.

[8] P. R. Lewis, A. Chandra, S. Parsons, E. Robinson, K. Glette, R. Bahsoon, $\mathrm{J}$. Torresen, and X. Yao, A survey of self-awareness and its application in computing systems, 2011.

[9] H. Hoffmann, M. Maggio, M. D. Santambrogio, A. Leva, and A. Agarwal, "Seec: A framework for self-aware computing," 2010.

[10] M. Maggio, T. Abdelzaher, and L. Esterle, Self-adaptation for Individual Self-aware Computing Systems. Springer International Publishing, 2017.

[11] A. Jantsch, N. Dutt, and A. M. Rahmani, "Self-Awareness in Systems on Chip-A Survey," IEEE Design \& Test, vol. 34, no. 6, pp. 8-26, 2017.

[12] A. Aminifar, Analysis, design, and optimization of embedded control systems. Linkoping University Electronic Press, 2016.

[13] A. Van de Vel, K. Cuppens, B. Bonroy, M. Milosevic, K. Jansen, S. Van Huffel, B. Vanrumste, P. Cras, L. Lagae, and B. Ceulemans, "Non-eeg seizure detection systems and potential sudep prevention: State of the art: Review and update," Seizure, vol. 41, pp. 141-153, 2016.

[14] M. J. Morrell, "Responsive cortical stimulation for the treatment of medically intractable partial epilepsy," Neurology, vol. 77, no. 13, pp. 1295-1304, 2011.

[15] S. Beniczky, I. Conradsen, O. Henning, M. Fabricius, and P. Wolf, "Automated real-time detection of tonic-clonic seizures using a wearable emg device," Neurology, vol. 90, no. 5, pp. e428-e434, 2018. 
[16] C. Á. Szabó, L. C. Morgan, K. M. Karkar, L. D. Leary, O. V. Lie, M. Girouard, and J. E. Cavazos, "Electromyography-based seizure detector: Preliminary results comparing a generalized tonic-clonic seizure detection algorithm to video-eeg recordings," Epilepsia, vol. 56, no. 9, pp. 1432-1437, 2015.

[17] O. Devinsky, K. Perrine, and W. H. Theodore, "Interictal autonomic nervous system function in patients with epilepsy," Epilepsia, vol. 35, no. 1, pp. 199-204, 1994.

[18] B. B. Wannamaker, "Autonomic nervous system and epilepsy," Epilepsia, vol. 26, pp. 31-39, 1985.

[19] K. Jansen and L. Lagae, "Cardiac changes in epilepsy," Seizure, vol. 19, no. 8, pp. $455-460,2010$

[20] C. Sevcencu and J. J. Struijk, "Autonomic alterations and cardiac changes in epilepsy," Epilepsia, vol. 51, no. 5, pp. 725-737, 2010.

[21] M. Zijlmans, D. Flanagan, and J. Gotman, "Heart rate changes and ecg abnormalities during epileptic seizures: prevalence and definition of an objective clinical sign," Epilepsia, vol. 43, no. 8, pp. 847-854, 2002.

[22] K. B. Nilsen, M. Haram, S. Tangedal, T. Sand, and E. Brodtkorb, "Is elevated pre-ictal heart rate associated with secondary generalization in partial epilepsy?" Seizure, vol. 19, no. 5, pp. 291-295, 2010.

[23] P. Boon, K. Vonck, K. van Rijckevorsel, R. El Tahry, C. E. Elger, N. Mullatti, A. Schulze-Bonhage, L. Wagner, B. Diehl, H. Hamer et al., "A prospective, multicenter study of cardiac-based seizure detection to activate vagus nerve stimulation," Seizure, vol. 32, pp. 52-61, 2015.

[24] S. V. Kothare and K. Singh, "Cardiorespiratory abnormalities during epileptic seizures," Sleep medicine, vol. 15, no. 12, pp. 1433-1439, 2014.

[25] O. Devinsky, "Effects of seizures on autonomic and cardiovascular function," Epilepsy currents, vol. 4, no. 2, pp. 43-46, 2004.

[26] D. Cogan, J. Birjandtalab, M. Nourani, J. Harvey, and V. Nagaraddi, "Multi-biosignal analysis for epileptic seizure monitoring," International journal of neural systems, vol. 27, no. 01, p. 1650031, 2017.

[27] M. E. O'Regan and J. K. Brown, "Abnormalities in cardiac and respiratory function observed during seizures in childhood," Developmental Medicine \& Child Neurology, vol. 47, no. 1, pp. 4-9, 2005.

[28] A. S. Blum, "Respiratory physiology of seizures," Journal of Clinical Neurophysiology, vol. 26, no. 5, pp. 309-315, 2009.

[29] M. Seyal, L. M. Bateman, and C.-S. Li, "Impact of periictal interventions on respiratory dysfunction, postictal eeg suppression, and postictal immobility," Epilepsia, vol. 54, no. 2, pp. 377-382, 2013.

[30] L. M. Bateman, M. Spitz, and M. Seyal, "Ictal hypoventilation contributes to cardiac arrhythmia and sudep: Report on two deaths in videoeeg-monitored patients," Epilepsia, vol. 51, no. 5, pp. 916-920, 2010.

[31] L. M. Bateman, C.-S. Li, T.-C. Lin, and M. Seyal, "Serotonin reuptake inhibitors are associated with reduced severity of ictal hypoxemia in medically refractory partial epilepsy," Epilepsia, vol. 51, no. 10, pp. 2211-2214, 2010

[32] M.-Z. Poh, T. Loddenkemper, N. C. Swenson, S. Goyal, J. R. Madsen, and R. W. Picard, "Continuous monitoring of electrodermal activity during epileptic seizures using a wearable sensor," in Engineering in Medicine and Biology Society (EMBC), 2010 Annual International Conference of the IEEE. IEEE, 2010, pp. 4415-4418.

[33] K. S. Eggleston, B. D. Olin, and R. S. Fisher, "Ictal tachycardia: the head-heart connection," Seizure, vol. 23, no. 7, pp. 496-505, 2014.

[34] K. Jansen, C. Varon, S. Van Huffel, and L. Lagae, "Peri-ictal ecg changes in childhood epilepsy: implications for detection systems," Epilepsy \& Behavior, vol. 29, no. 1, pp. 72-76, 2013.

[35] K. Schiecke, M. Wacker, D. Piper, F. Benninger, M. Feucht, and H. Witte, "Time-variant, frequency-selective, linear and nonlinear analysis of heart rate variability in children with temporal lobe epilepsy," IEEE Transactions on Biomedical Engineering, vol. 61, no. 6, pp. 17981808,2014

[36] F. Forooghifar, A. Aminifar, and D. Atienza, "Self-aware wearable systems in epileptic seizure detection," in Proceedings of Euromicro Conference on Digital System Design (DSD) 2018. IEEE, 2018.

[37] S. Murali, F. Rincon, and D. Atienza, "A wearable device for physical and emotional health monitoring," in Computing in Cardiology Conference (CinC), 2015. IEEE, 2015, pp. 121-124.

[38] LivaNova. (2016) Vagus nerve stimulation (vns therapy). [Online] Available: http://www.livanova.cyberonics.com

[39] AspireSR. (2016) Vagus nerve stimulation (vns therapy). [Online]. Available: https://www.epilepsy.com/learn/treating-seizuresand-epilepsy/devices/vagus-nerve-stimulation-vns

[40] C. Hoppe, M. Feldmann, B. Blachut, R. Surges, C. E. Elger, and C. Helmstaedter, "Novel techniques for automated seizure registration: patients' wants and needs," Epilepsy \& Behavior, vol. 52, pp. 1-7, 2015

[41] D. Wang, D. Ren, K. Li, Y. Feng, D. Ma, X. Yan, and G. Wang, "Epileptic seizure detection in long-term eeg recordings by using wavelet- based directed transfer function," IEEE Transactions on Biomedical Engineering, 2018.

[42] L. Guo, D. Rivero, and A. Pazos, "Epileptic seizure detection using multiwavelet transform based approximate entropy and artificial neural networks," Journal of neuroscience methods, vol. 193, no. 1, pp. 156163,2010

[43] S. Raghu, N. Sriraam, G. P. Kumar, and A. Hegde, "A novel approach for real time recognition of epileptic seizures using minimum variance modified fuzzy entropy," IEEE Transactions on Biomedical Engineering, 2018.

[44] K. Fu, J. Qu, Y. Chai, and T. Zou, "Hilbert marginal spectrum analysis for automatic seizure detection in eeg signals," Biomedical Signal Processing and Control, vol. 18, pp. 179-185, 2015.

[45] J.-L. Song, W. Hu, and R. Zhang, "Automated detection of epileptic eegs using a novel fusion feature and extreme learning machine," Neurocomputing, vol. 175, pp. 383-391, 2016.

[46] A. R. Hassan, S. Siuly, and Y. Zhang, "Epileptic seizure detection in eeg signals using tunable-q factor wavelet transform and bootstrap aggregating," Computer methods and programs in biomedicine, vol. 137, pp. 247-259, 2016.

[47] M. Ravan, S. Sabesan, and D. O'Neill, "On quantitative biomarkers of vns therapy using eeg and ecg signals," IEEE Transactions on Biomedical Engineering, vol. 64, no. 2, pp. 419-428, 2017.

[48] D. Pascual, Aminifar, and D. Atienza, "A self-learning methodology for epileptic seizure detection with minimally supervised edge labeling," in Design, Automation \& Test in Europe Conference \& Exhibition (DATE), 2019. IEEE, 2019.

[49] E. Foundation. (2016) Sami. [Online]. Available: https://www.samialert.com

[50] Epi-Watcher. (2017) Epi-watcher. [Online]. Available: http://www.vahlkamp.nl

[51] A. P. Narechania, I. I. Garic, I. Sen-Gupta, M. P. Macken, E. E. Gerard, and S. U. Schuele, "Assessment of a quasi-piezoelectric mattress monitor as a detection system for generalized convulsions," Epilepsy \& Behavior, vol. 28, no. 2, pp. 172-176, 2013.

[52] S. Monitor. (2016) Smartwatch. [Online]. Available: http://smartmonitor.com/

[53] Empatica. (2016) Embrace alert system. [Online]. Available: https://www.empatica.com/embrace-watch-epilepsy-monitor

[54] S. N. Larsen, I. Conradsen, S. Beniczky, and H. B. Sorensen, "Detection of tonic epileptic seizures based on surface electromyography," in Engineering in Medicine and Biology Society (EMBC), 2014 36th Annual International Conference of the IEEE. IEEE, 2014, pp. 942-945.

[55] C. A. Szabo, L. C. Morgan, K. M. Karkar, L. D. Leary, O. V. Lie, M. Girouard, and J. E. Cavazos, "Electromyography-based seizure detector: Preliminary results comparing a generalized tonic-clonic seizure detection algorithm to video-eeg recordings," Epilepsia, vol. 56, no. 9, pp. 1432-1437, 2015.

[56] W. J. Van Elmpt, T. M. Nijsen, P. A. Griep, and J. B. Arends, "A model of heart rate changes to detect seizures in severe epilepsy," Seizure, vol. 15 , no. 6 , pp. 366-375, 2006.

[57] O. Doyle, A. Temko, W. Marnane, G. Lightbody, and G. Boylan, "Heart rate based automatic seizure detection in the newborn," Medical engineering \& physics, vol. 32, no. 8, pp. 829-839, 2010.

[58] K. Vandecasteele, T. De Cooman, Y. Gu, E. Cleeren, K. Claes, W. V. Paesschen, S. V. Huffel, and B. Hunyadi, "Automated epileptic seizure detection based on wearable ecg and ppg in a hospital environment," Sensors, vol. 17, no. 10, p. 2338, 2017.

[59] F. Masse, M. V. Bussel, A. Serteyn, J. Arends, and J. Penders, "Miniaturized wireless ecg monitor for real-time detection of epileptic seizures," ACM Transactions on Embedded Computing Systems (TECS), vol. 12, no. 4, p. 102, 2013

[60] C. Ungureanu, V. Bui, W. Roosmalen, R. M. Aarts, J. B. Arends, R. Verhoeven, and J. J. Lukkien, "A wearable monitoring system for nocturnal epileptic seizures," in Medical Information and Communication Technology (ISMICT), 2014 8th International Symposium on. IEEE, 2014, pp. 1-5.

[61] C. Varon, K. Jansen, L. Lagae, and S. Van Huffel, "Detection of epileptic seizures by means of morphological changes in the ecg," in Computing in Cardiology Conference (CinC), 2013. IEEE, 2013, pp. 863-866.

[62] I. Osorio, "Automated seizure detection using ekg," International journal of neural systems, vol. 24, no. 02, p. 1450001, 2014.

[63] J. Jeppesen, S. Beniczky, P. Johansen, P. Sidenius, and A. FuglsangFrederiksen, "Using lorenz plot and cardiac sympathetic index of heart rate variability for detecting seizures for patients with epilepsy," in the 36th IEEE Annual International Conference of Engineering in Medicine and Biology Society (EMBC). IEEE, 2014, pp. 4563-4566. 
[64] — "Detection of epileptic seizures with a modified heart rate variability algorithm based on lorenz plot," Seizure, vol. 24, pp. 1-7, 2015.

[65] J. Pavei, R. G. Heinzen, B. Novakova, R. Walz, A. J. Serra, M. Reuber, A. Ponnusamy, and J. L. Marques, "Early seizure detection based on cardiac autonomic regulation dynamics," Frontiers in Physiology, vol. 8 , p. 765, 2017.

[66] N. Dutt, A. Jantsch, and S. Sarma, "Toward smart embedded systems: A self-aware system-on-chip (soc) perspective," ACM Transactions on Embedded Computing Systems (TECS), vol. 15, no. 2, p. 22, 2016.

[67] J. S. Preden, K. Tammemae, A. Jantsch, M. Leier, A. Riid, and E. Calis, "The benefits of self-awareness and attention in fog and mist computing," Computer, vol. 48, no. 7, pp. 37-45, 2015.

[68] T. Chen, F. Faniyi, R. Bahsoon, P. R. Lewis, X. Yao, L. L. Minku, and L. Esterle, "The handbook of engineering self-aware and self-expressive systems," arXiv preprint arXiv:1409.1793, 2014

[69] F. Faniyi, P. R. Lewis, R. Bahsoon, and X. Yao, "Architecting self-aware software systems," in Software Architecture (WICSA), 2014 IEEE/IFIP Conference on. IEEE, 2014, pp. 91-94.

[70] A. Aminifar, P. Tabuada, P. Eles, and Z. Peng, "Self-triggered controllers and hard real-time guarantees," in Proceedings of the 2016 Conference on Design, Automation \& Test in Europe. EDA Consortium, 2016, pp. 636-641.

[71] A. Aminifar, "Self-triggered controllers, resource sharing, and hard guarantees," in 2016 Second International Conference on Event-based Control, Communication, and Signal Processing (EBCCSP). IEEE, 2016, pp. 1-7.

[72] A. Anzanpour, I. Azimi, M. Gotzinger, A. M. Rahmani, N. TaheriNejad, P. Liljeberg, A. Jantsch, and N. Dutt, "Self-awareness in remote health monitoring systems using wearable electronics," in Proceedings of the Conference on Design, Automation \& Test in Europe. European Design and Automation Association, 2017, pp. 1056-1061.

[73] N. TaheriNejad, A. Jantsch, and D. Pollreisz, "Comprehensive observation and its role in self-awareness; an emotion recognition system example," Self, vol. 11, p. 1, 2016.

[74] L. Breiman, "Random forests," Machine learning, vol. 45, no. 1, pp. 5-32, 2001.

[75] J. Pan and W. J. Tompkins, "A real-time qrs detection algorithm," IEEE Transactions on Biomedical Engineering, vol. BME-32, no. 3, pp. 230236, 1985
[76] R. Thompson, "A note on restricted maximum likelihood estimation with an alternative outlier model," Journal of the Royal Statistical Society. Series B (Methodological), pp. 53-55, 1985.

[77] P. C. Ivanov, L. A. N. Amaral, A. L. Goldberger, S. Havlin, M. G. Rosenblum, Z. R. Struzik, and H. E. Stanley, "Multifractality in human heartbeat dynamics," Nature, vol. 399, no. 6735, p. 461, 1999.

[78] P. de Chazal, C. Heneghan, E. Sheridan, R. Reilly, P. Nolan, and M. O’Malley, "Automated processing of the single-lead electrocardiogram for the detection of obstructive sleep apnoea," IEEE Transactions on Biomedical Engineering, vol. 50, no. 6, pp. 686-696, 2003.

[79] H. Ocak, "Automatic detection of epileptic seizures in eeg using discrete wavelet transform and approximate entropy," Expert Systems with Applications, vol. 36, no. 2, pp. 2027-2036, 2009.

[80] X. Chen, I. C. Solomon, and K. H. Chon, "Comparison of the use of approximate entropy and sample entropy: applications to neural respiratory signal," in Engineering in Medicine and Biology Society, 2005. IEEE-EMBS 2005. 27th Annual International Conference of the IEEE, 2006, pp. 4212-4215.

[81] C. Bandt and B. Pompe, "Permutation entropy: a natural complexity measure for time series," Physical review letters, vol. 88, no. 17, p. 174102,2002

[82] U. R. Acharya, H. Fujita, V. K. Sudarshan, S. Bhat, and J. E. Koh, "Application of entropies for automated diagnosis of epilepsy using eeg signals: A review," Knowledge-Based Systems, vol. 88, pp. 85-96, 2015.

[83] Scargle and D. Jeffrey, "Studies in astronomical time series analysis. ii. statistical aspects of spectral analysis of unevenly spaced data," Astrophysical Journal, vol. 263, pp. 835-853, 1982.

[84] Lomb and R. Nicholas, "Least-squares frequency analysis of unequally spaced data," Astrophysics and Space Science, vol. 39, pp. 447-462, 1976.

[85] P. J. Fleming and J. J. Wallace, "How not to lie with statistics: the correct way to summarize benchmark results," Communications of the $A C M$, vol. 29 , no. 3, pp. 218-221, 1986.

[86] STM32L151RD, Ultra-low-power ARM Cortex-M3 MCU with 384 Kbytes Flash, $32 \mathrm{MHz}$ CPU, USB, 3xOp-amp - STMicroelectronics. [87] ECG electrode for sensitive skin, Ambu BlueSensor VLC.

[88] T. Instruments. (2018) Ads7142. [Online]. Available: http://www.ti.com/product/ADS7142/description 